\title{
Composition and yield of non-cellulosic and cellulosic sugars in soluble and particulate fractions during consolidated bioprocessing of poplar biomass by Clostridium thermocellum
}

\author{
Ajaya K. Biswal \\ University of Georgia \\ Neal N. Hengge \\ National Renewable Energy Laboratory \\ lan M. Black \\ University of Georgia \\ Melani A. Atmodjo \\ University of Georgia \\ Sushree S. Mohanty \\ University of Georgia \\ David Ryno \\ University of Georgia \\ Michael E. Himmel \\ National Renewable Energy Laboratory \\ Parastoo Azadi \\ University of Georgia \\ Yannick J. Bomble \\ National Renewable Energy Laboratory \\ Debra Mohnen ( $\nabla$ dmohnen@ccrc.uga.edu ) \\ University of Georgia
}

\section{Research Article}

Keywords: Consolidated bioprocessing, Populus, Clostridium thermocellum, Cellulose, Lignin, Pectin, Hemicellulose (Xylan), Non-cellulosic wall polysaccharides

Posted Date: October 25th, 2021

DOI: https://doi.org/10.21203/rs.3.rs-991856/v1 
License: (c) (i) This work is licensed under a Creative Commons Attribution 4.0 International License. Read Full License

Version of Record: A version of this preprint was published at Biotechnology for Biofuels and Bioproducts on February 28th, 2022. See the published version at https://doi.org/10.1186/s13068-022-02119-9. 


\section{Abstract \\ Background}

Terrestrial plant biomass is the primary renewable carbon feedstock for enabling transition to a sustainable bioeconomy. Consolidated bioprocessing (CBP) by the cellulolytic thermophile Clostridium thermocellum offers a single step microbial platform for production of biofuels and biochemicals via simultaneous solubilization of carbohydrates from lignocellulosic biomass and conversion to products. Here, solubilization of cell wall cellulosic, hemicellulosic, and pectic polysaccharides in the liquor and solid residues generated during $\mathrm{CBP}$ of poplar biomass by $\mathrm{C}$. thermocellum was analyzed.

\section{Results}

The total amount of biomass solubilized in the $C$. thermocellum fermentation platform was $5.8,10.3$, and $13.7 \%$ of milled non-pretreated poplar after 24,48 , and $120 \mathrm{~h}$, respectively. When combined, these results demonstrate solubilization of $24 \%$ cellulose and $17 \%$ non-cellulosic sugars after $120 \mathrm{~h}$, consistent with prior reports. The solubilization of non-cellulosic sugars by $C$. thermocellum compared to uninoculated control fermentations was 13 to $36 \%$ of arabinose (Ara), xylose (Xyl), galactose (Gal), mannose (Man), and glucose (Glc); and 15\% and 3\% of fucose and glucuronic acid, respectively. No rhamnose was solubilized and $71 \%$ of the galacturonic acid (GalA) was solubilized. These results indicate that $C$. thermocellum may be selective for the types and/or rate of solubilization of the non-cellulosic wall polymers. Xyl, Man, and Glc were found to accumulate in the fermentation liquor at levels greater than in uninoculated control fermentations, whereas Ara and Gal did not accumulate, suggesting that $C$. thermocellum solubilizes both hemicelluloses and pectins but utilizes them differently. After five days of fermentation, the amount of Rha in the solid residues increased $27 \%$, indicating that the Rha-containing polymers (i.e., rhamnogalacturonan I, RG-I) were not effectively solubilized by $C$. thermocellum CBP. Comparison of the sugars in the liquor versus solid residue showed that $C$. thermocellum solubilized hemicellulosic xylan and mannan but did not fully utilize them, solubilized and appeared to utilize pectic homogalacturonan, and did not solubilize pectic RG-I.

\section{Conclusions}

The significant increase in Rha in poplar solid residues following CBP indicates that $C$. thermocellum did not solubilize RG-I. These results support the hypothesis that this pectic glycan may be a barrier for efficient solubilization of poplar by $C$. thermocellum.

\section{Background}

We are at an inflection point in which a resource depletion, fossil fuel-based economy must be replaced by a sustainable carbon negative bioeconomy $[1,2]$. This transition includes the use of biomass as the 
major carbohydrate-rich renewable resource for production of transportation fuels, chemicals, and materials. Dedicated cellulosic feedstocks, such as trees and herbaceous grasses, along with residues from agricultural, forest restoration, and municipal solid waste are available at the scales needed for this transition. The development of large scale and cost effective processing technologies to convert the structurally complex plant biomass to bioproducts, however, remains challenging [3].

The bulk of carbohydrate in dedicated cellulosic feedstock is comprised of cellulose microfibrils, hemicelluloses, and lignin; with lesser amounts of pectin and glycoproteins [4-7]. The primary, secondary, tertiary, and quaternary structures of these components and their final associations and architecture in the cell wall have evolved to vary by cell type and developmental state, thus providing sessile plants and their cells structural and functional properties that enable survival in harsh biotic and abiotic environments. The primary challenge for the use of plant biomass as a resource for the production of biofuels, chemicals and biocommodities is therefore the development of processing strategies that overcome natural recalcitrance to deconstruction and provide high yields of the desired sugars for fermentation $[1,3]$.

While transportation fuel for light duty vehicles is expected to be replaced by electric energy, liquid fuel will be required into the foreseeable future for aviation and marine transportation. Thus, efforts to produce liquid fuel from biomass remain critical. Multiple biomass processing and fermentation strategies to obtain $\mathrm{C} 5$ and $\mathrm{C} 6$ sugars from lignocellulosic biomass have been developed, including thermal, chemical, biochemical and microbial processes [3]. However, none have proven sufficiently cost effective to yield a thriving biofuel industry due to hurdles that include the cost of pretreatment to "loosen" biomass structure and allow hydrolytic enzymes to gain access to cell wall polymers, the cost of exogenous enzymes needed in classical simultaneous saccharification and fermentation (SSF), and the production of inhibitors of the fermentation process during biomass pretreatment. An approach that circumvents some of these obstacles is consolidated bioprocessing (CBP), in which a suitable microorganism can both produce enzymes able to solubilize sugars from the untreated biomass and ferment the sugars to biofuels, providing a single step process. An optimized CBP platform would eliminate the need for exogenous enzymes and pretreatment, improve efficiency, and decrease capital investment, steps important for development of a cost competitive process $[1,3]$.

A leading microbial candidate for CBP is the anaerobic Gram positive thermophile, Clostridium thermocellum, which is one of the most effective crystalline cellulose utilizing microbes found in Nature [3]. CBP using $C$. thermocellum offers distinct process advantages, including reduced risk of microbial contamination and increased solubilization of biomass polymers due to high growth temperatures, facile integration into existing industrial processing schemes due to reduced cooling requirements, and the production of highly efficient cellulosomes which provide unique solubilization properties on crystalline cellulose [3]. C. thermocellum can hydrolyze all three types of cell wall polymers, cellulose, hemicellulose (e.g., xylan) and pectin; the latter being a major component of the "glue" that holds the cells of plant biomass together [8-10]. Limitations in the use of $C$. thermocellum for the production of biofuels include the inability of native strains to utilize $\mathrm{C} 5$ sugars and a relatively low tolerance to ethanol [11]. 
Although $C$. thermocellum is very efficient at hydrolyzing and utilizing cellulose, and produces enzymes that solubilize the other two classes of non-cellulosic cell wall polysaccharides, hemicellulose and pectin, it has been reported that $C$. thermocellum cannot utilize the noncellulosic polymers for growth [11]. Numerous studies have confirmed that wild type $C$. thermocellum can hydrolyze xylan, the most abundant non-cellulosic polysaccharide in grasses and hardwood feedstocks, but cannot utilize xylose or arabinose for growth. There are fewer published studies on the metabolism of pectin by $C$. thermocellum and those that are available are contradictory. Aburaya and coworkers [12] reported that $C$. thermocellum cannot grow on pectin, whereas Spinnler and coworkers [13] showed that although $C$. thermocellum could not grow on monogalacturonic acid, a major glycosyl residue of pectin, it did grow well on polygalacturonic acid and sugar beet pectin. These contradictory results leave open the question as to whether or not $C$. thermocellum utilizes pectin.

C. thermocellum is highly effective in solubilizing pure crystalline cellulose, with reports of up to $100 \%$ solubilization of Avicel [3]. The solubilization of the cellulose and non-cellulolosic cell wall polymers in plant biomass by $C$. thermocellum is more difficult due to the heterogeneous nature of these polymers and their homo- and hetero-associations. The degrees of solubilization of biomass by $C$. thermocellum depends on the type of biomass, severity of the pretreatment, and methods used to quanitify carbohydrate yields. For example, total carbohydrate fractional yields of $20-31 \%$ and $32-45 \%$ have been reported for $C$. thermocellum solubilization of milled non-pretreated poplar and switchgrass biomass, respectively [14]. Pretreatment of biomass can increase solubilization rates and amounts dramatically; however, these processes require additional energy and processing steps and therefore increase process cost. The methods generally used to calculate mass fraction solubilization are based on fractional glucose, xylose, and arabinose yields following microbial growth and fermentation. In such calculations, changes in the other non-cellulose sugars are typically not monitored since they represent only a small fraction of the sugars in the dedicated grass and woody feedstock. For example, galacturonic acid, the major pectic sugar, accounts for only $~ 6 \%$ of poplar wood and $1 \%$ of switchgrass tillers [15]. However, although a minor component, changes in the amount and structure of pectin have been shown to increase both grass and woody biomass yield and to make the biomass more amenable to enzymatic deconstruction, due to the role of pectin in polymer/polymer and thus cell/cell adhesion in the wall [4]. Thus, knowledge of the efficiency of $C$. thermocellum in solubilizing and utilizing pectin during CBP is important for understanding and potentially improving the CBP process.

The goal of this study was to better understand the solubilization of cellulose and non-cellulosic sugars from milled, non-pretreated Center for Bioenergy Innovation (CBI) reference poplar [16] during CBP by $C$. thermocellum. Determination of cellulosic and non-cellulosic sugar mass yields in both the fermentation broth and residues during CBP by $C$. thermocellum was carried out to identify potential cell wall structural and architectural changes and limitations to solubilization of non-pretreated poplar biomass. The results of this study provide information about non-cellulosic sugars and likely corresponding polymers that are not effectively solubilized from milled, non-pretreated poplar by $C$. thermocellum. This study also 
provides a foundation for comparisons of the efficacy of $C$. thermocellum solubilization of biomass from mutants and variants with modified cell wall and yield properties; as well as the effects of different biomass pretreatment strategies on the ability of $C$. thermocellum to solubilize biomass at different biomass loadings.

\section{Results}

\section{Solubilization of poplar biomass using C. thermocellum.}

A schematic of the microbial fermentation and analysis procedure used to evaluate the solubilization of poplar biomass by C. thermocellum DSM1313 is shown in Additional File 1: Fig. S1. Fermentations (0.5 $\mathrm{L}$ ) were performed with $5.68 \mathrm{~g}$ chipped and milled poplar biomass at $5 \mathrm{~g} / \mathrm{L}$ total glucan loading corresponding to approximately $11.4 \mathrm{~g} / \mathrm{L}$ total solids loading. Changes in total mass yield and cellulosic and non-cellulosic sugar composition in the liquid and solid fractions were measured at times zero, 24, 48

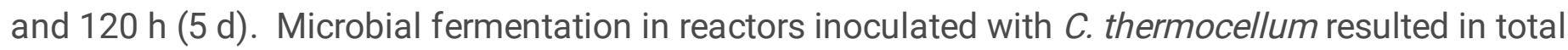
solids solubilization of approximately $5.8 \%, 10.3 \%$, and $13.7 \%$ after 24,48 , and 120 h, respectively, while control reactors without the microbe (henceforth called fermentation controls) resulted in $4.5 \%, 4.6 \%$ and $6.3 \%$ total solids solubilization at the same time points (Fig. 1 and Additional File 2: Fig. S2). The total amount of biomass solubilized specifically by $C$. thermocellum CBP was calculated by subtracting the end-point biomass weight recovered after CBP fermentation from the starting biomass, as described in the material and methods, and substracting from this the amount of biomass solubilized by the fermentation controls which did not contain the microbe. When the amount of biomass solubilized due to the heat and medium conditions in the reactors (i.e., as in the fermentation controls) was subtracted, the results showed that $C$. thermocellum CBP solubilized $1.3 \%, 5.7 \%$ and $7.4 \%$ of the poplar biomass after 24 , 48 , and $120 \mathrm{~h}$, respectively.

\section{Evaluation of dry mass content and glycosyl residue composition of post fermentation liquor from $C$. thermocellum CBP fermentations}

After 24,48 , and $120 \mathrm{~h}$ of microbial and control fermentations, the content of each fermentation reactor was centrifuged to separate the liquor (i.e., the supernatant representing the soluble fraction) from the solid residue. The CBP and fermentation control liquor samples were lyophilized for five days and the dry mass was recorded. There was a significant increase (16\% to $23 \%)$ in the dry mass recovered from the CBP liquor compared to the controls at 24,48 , and 120 h, respectively (Fig. 2). These results showed that C. thermocellum solubilized biomass over the course of the fermentations. Since the amount of dry mass recovered from the liquor of the fermentation controls and the CBP fermentations was greater than the amount of total solids solubilized from the biomass (Fig. 2 and Additional File 2: Fig. S2), these results suggested that the additional mass recovered in the control liquor may have resulted from dried culture medium components and that the additional mass recovered in the $C$. thermocellum fermentations may have resulted from the culture medium components plus microbe culture components. 
In order to determine which components of the poplar biomass were solubilized by $C$. thermocellum and recovered in the liquor during the CBP fermentations, the liquor samples from the control and $C$. thermocellum fermentations were analyzed for glycosyl residue composition by trimethylsilyl (TMS) derivatization and GC-MS. This method specifically quantifies the non-cellulosic sugar composition [15]. Noteworthy, a large amount of non-cellulosic sugars were present in the fermentation controls, indicating that some of the non-cellulosic polysaccharides in the poplar biomass were solubilized by the temperature conditions and medium used during the fermentation. Significantly more sugars; however, were solubilized from the poplar biomass by $C$. thermocellum fermentations compared to the controls including $41 \%$ to $267 \%$ more xylose (Xyl), $35 \%$ to $80 \%$ more mannose (Man), and $19 \%$ to $35 \%$ more glucose (GIC) over the $120 \mathrm{~h}$ fermentation (Fig. 3 and Additional File 3: Fig. S3). The accumulation of these sugars in the liquor suggested that $C$. thermocellum was able to solubilize hemicelluloses, such as glucuronoxylan and glucomannan in poplar wood but did not completely take up or metabolize these sugars. Only trace amounts of acidic sugars (e.g., galacturonic acid, GalA) were detected in the liquid fractions.

Interestingly, arabinose (Ara), rhamnose (Rha), and galactose (Gal) were also detected in the liquor of both the control and $C$. thermocellulum fermentations. However, their levels were significantly less in the liquor from the CBP fermentations compared to the liquor from the fermentation controls, being 36 to $57 \%$ less for Ara, 24 to $49 \%$ less for Rha, and 32 to $46 \%$ less for Gal at $48 \mathrm{~h}$ and $120 \mathrm{~h}$ ). The reduction in the levels of these sugars in the liquor of the $C$. thermocellum fermentations compared to the control fermentations, suggested that $C$. thermocellum may have assimilated these sugars. However, since the levels of these sugars were greater in the control liquor than in the CPB liquor, it was not possible from these data alone to conclude whether or not $C$. thermocellum had solubilized these sugars from the biomass, or rather they had all resulted from the process conditions. To determine how much sugar $C$. thermocellum was able to solubilize from the biomass, the solid residues obtained during the fermentations were also evaluated.

\section{Production of alcohol insoluble residues from poplar residual biomass and characterization of lignin, cellulose, and non-cellulosic sugars in the residual biomass}

In order to determine the amount of lignin, cellulose and non-cellulosic polysaccharides remaining in fermentation residues during $C$. thermocellum $\mathrm{CBP}$, alcohol insoluble residue (AIR) was produced from the post-fermentation biomass (solid residues) remaining after $\mathrm{CBP}$ and in the fermentation controls. The resulting AIR, which represents cell walls, was de-starched for $48 \mathrm{~h}$ with a-amylase and the resulting destarched AIR was analyzed for cellulose, lignin and non-cellulosic sugars. The yield of total AIR per gram of solid residue from the fermentation controls and the $C$. thermocellum CBP fermentationsafter the first $24 \mathrm{~h}$ were comparable (Additional File 4: Fig. S4). However, the yield of AIR per gram of solid residue from the CBP solid residuals was $3 \%$ and $5 \%$ less than that from the controls after 48 and $120 \mathrm{~h}$ incubation, respectively. This slightly lower yield of AIR per gram solid residue in the $C$. thermocellum CBPfermentations may have been due to components contributed to the solid residues 
by $C$. thermocellum over the course of the fermentation that were not recovered by the ethanol and chloroform/methanol extraction process used for AIR preparation.

\section{Lignin content}

To evaluate the amount and composition of lignin in the post-fermentation solid residues of poplar wood, the amount of total lignin, guaiacyl $(\mathrm{G})$, p-hydroxyphenyl $(\mathrm{H})$ and syringyl $(\mathrm{S})$ lignin subunits and the lignin S/G ratio were measured by pyrolysis molecular beam mass spectrometry (py-MBMS) [4]. The total lignin content of the $\mathrm{CBI}$ reference poplar was significantly less (14\%) than the total lignin content of the BESC standard poplar (Fig. 4A and Additional File 5: Fig. S5A). The CBI reference poplar line (GW-9947) was previously identified as a low recalcitrance poplar natural variant with reduced lignin and a high syringyl-to-guaiacyl (S/G) ratio [16], a difference confirmed by the py-MBMS lignin analysis results reported here.

Analysis of the lignin content of the $\mathrm{CBI}$ reference poplar biomass versus the lignin content in the solid residues of the $24 \mathrm{~h}, 48 \mathrm{~h}$, and $120 \mathrm{~h}$ CBP fermentation controls revealed an unexpected reduction in the amount of total, $\mathrm{S}$, and $\mathrm{G}$ lignin, suggesting that some lignin-containing wall material is solubilized during the process used for fermentation. The total, $\mathrm{S}$, and $\mathrm{G}$ lignin content of the CBP solid residual samples following fermentation of the $\mathrm{CBI}$ reference poplar by $C$. thermocellum increased on average $25 \%, 24 \%$, and $13 \%$ (per mg starting biomass dry weight), respectively, compared to the fermentation controls (Fig. 4A-D and Additional File 5: Fig. S5), whereas no major changes were observed in the $\mathrm{H}$ subunit content. The increase in the percentage of lignin in the residual biomass during CBP fermentation by $C$. thermocellum was at least partially associated with the solubilization of hemicellulose and cellulose (see below), leading to a greater weight percentage of total lignin in the residues.

The lignin S/G ratios were significantly increased (6 to 14\%) in CBP solid residue compared to those of controls (Fig. 4E). A higher S/G ratio in the residual solids following $C$. thermocellum $\mathrm{CBP}$ fermentation of poplar wood has been previously described [14, 16-18]. One hypothesis for this result is that some of the hemicellulose or cellulose solubilized by $C$. thermocellum is associated with lignin that has a higher $\mathrm{G} / \mathrm{S}$ ratio, which was preferentially solubilized along with the glycans. There were no major changes in the C5:C6 ratio of poplar solid residues in the control or $C$. thermocellum CBP fermentations (Fig. 4F).

\section{Cellulose content}

Two methods were used to determine the cellulose content in the post-fermentation solid residues. The first method involved measuring the amount of glucose present in non-crystalline polysaccharides (i.e., non-cellulosic cell wall glycans) by GC-MS of TMS-derivatized methyl glycosides and substracting this value from the total cellulosic plus non-cellulosic glucose as obtained by Saeman (1945) hydrolysis of crystalline cellulose $[19,20]$ followed by glycosyl residue composition analysis (Additional File 6: Fig. S6A-C). This measurement of cellulose (Fig. 5A and Additional File 6: Fig. S6C) was in good agreement

with published values of cellulose content for poplar wood [21, 22]. The second method used to measure cellulose content was the recently developed fully methylated alditol (MA) procedure which enables 
analysis of insoluble polysaccharides through their permethylation in DMSO to produce methyl alditol derivatives that can be analyzed by GC-MS [23] (Fig. 5B and Additional File 6: Fig. S6D-F). The MA method thus also provides a measure of total Glc which, upon substracting the amount of non-cellulosic Glc (see subsection below), provides the cellulose content. CBP fermentation of CBI reference poplar biomass for $5 \mathrm{~d}$ by $C$. thermocellum resulted in a significant $25 \%$ total reduction in the cellulose content (mg Glc/starting biomass) based on the Saeman hydrolysis method, which corresponded to a $21 \%$ reduction after subtracting the fermentation controls (Fig. 5A and Additional File 6: Fig. S6A-C). The MA method indicated a $29 \%$ total reduction in cellulose (mg Glc/starting biomass) and $25 \%$ reduction after subtracting the fermentation controls (Fig. 5B, Additional File 6: Fig. S6 D-F, Table 1). These values are comparable to those of Dumitrache and coworkers [21] who showed a $28 \%$ reduction in total glucose content of $P$. deltoides biomass after incubation of $C$. thermocellum ATCC27405 for $92 \mathrm{~h}$ at $60^{\circ} \mathrm{C}$ and confirmed that the microbe can solubilize a portion of the cellulose in non-pretreated poplar woody biomass.

\section{Glycosyl residue composition of non-cellulosic sugars in solid residues}

To study the solubilization of non-cellulosic cell wall glycans by $C$. thermocellum $\mathrm{CBP}$, the glycosyl residue composition of $\mathrm{CB}$ I reference poplar solid residues after 24,48 , and $120 \mathrm{~h}$ of microbial hydrolysis was measured and compared to those of the solid residues in the fermentation controls (Fig. 6). Glycosyl residue composition analysis of the solid residues before and after $C$. thermocellulm CBP revealed that the $\mathrm{mg}$ per mass starting biomass of six sugars was significantly decreased in the presence of $C$. thermocellum (Fig.6) with7\% to $14 \%$ decreased Xyl, $13 \%$ to $36 \%$ decreased Man, $13 \%$ to $23 \%$ decreased Glc, and $56 \%$ to $71 \%$ decreased GalA from $24 \mathrm{~h}$ to $120 \mathrm{~h}$. There was also $9 \%$ to $13 \%$ decreased Gal at $48 \mathrm{~h}$ to $120 \mathrm{~h}$ and $15 \%$ decreased Ara at $120 \mathrm{~h}$ indicating hydrolysis by $C$. thermocellum. Similar relative changes in post-fermentation glucose content (18\% decreased Glc/gram solid) has been reported for other high S/G woody phenotypes [17]. The particularly large reduction in the amount of GalA in the solid residue but little GalA in the liquor suggests the possible utilization of pectic sugars by $C$. thermocellum. Conversely, there was a significant trend for increased Rha (14\% to $27 \%$ ) in the CBP solid residues compared to the fermentation controls, suggesting that Rha containing polymers in the wall (e.g., RG-I) were not being solubilized and thus were found to increase in the final residue recovered. Although there was a small reduction in the total non-cellulosic sugars in the solid residue from the fermentation control, the $10 \%$ to $18 \%$ greater reduction in total non-cellulosic sugars in the solid residues following CBP fermentation by $C$. thermocellum indicates that the microbe solubilizes at least some of the non-cellulosic wall polymers including xylans and homogalacturonan (Fig. 6 and Additional File 7: Fig. S7).

A comparison of the amount of lignin, cellulose, and non-cellulosic sugars in the solid residues recovered over the $120 \mathrm{~h}$ fermentation (Fig. 7) shows that $24 \%$ percent of the cellulose was solubilized over $5 \mathrm{~d}$ of CBP fermentation by $C$. thermocellum (Fig. 7A, C). Simultaneouly, $17 \%$ of the non-cellulosic sugars were solubilized suggesting that $C$. thermocellum hydrolyzes these sugars during fermentation (Fig. 7B). The negative value of mass and percentage of lignin solubilization is due to the increase in the lignin content in the residual biomass as the cellulose and non-cellulosic sugars are solubilized (Fig. 7C and Table 1). 
An analysis of the percentage of the specific sugars solubilized (Fig. 7D and Table 1) reveals that Rha was the only sugar that was not solubilized by $C$. thermocellum during the fermentation (-21\% compared to starting biomass, Fig. 7D; $-27 \%$ compared to fermentation control, Table 1). This result suggests that Rha-containing cell wall polymers (i.e. RG-I) may be a key determinant limiting solubilization of poplar biomass during $\mathrm{CBP}$ fermentation by $\mathrm{C}$. thermocellum.

\section{Discussion}

C. thermocellum secretes an extensive array of glycoside hydrolases with more than 70 types of cellulosomal proteins capable of hydrolyzing different types of lignocellulosic biomass [24-27]. However, without pretreatment of the lignocellulosic biomass, this cellulolytic microbe cannot completely depolymerize the native complex polysaccharides in the plant cell wall of either woody and mature grassy feedstocks. The objective of this study was to characterize the solubilization of both cellulose and non-cellulosic sugars from non-pretreated milled CBI reference poplar biomass [16] by $C$. thermocellum by evaluating the starting biomass, the post-fermentation liquors, and solid residues recovered during the CBP process. Our goal was to quantify the amount of cellulosic and non-cellulosic sugars solubilized during the CBP process to provide information about which polymers in woody biomass $C$. thermocellum solubilizes and to identify potential limiting factors that could inform the development of an economically viable consolidated bioprocess.

C. thermocellum can efficiently solubilize crystalline cellulose, with reports of $95 \%$ and $93 \%$ solublization of Avicel at low $\left(5 \mathrm{~g} \mathrm{~L}^{-1}\right)$ [28] and high (100 $\left.\mathrm{g} \mathrm{L}^{-1}\right)$ [29] solids loadings, respectively. However, it has been reported to achieve a much lower $17 \%$ solubilization of woody Populus biomass at low ( $10 \mathrm{~g} \mathrm{~L}^{-1}$ dry weight, DW) solids loading and the solubilization was affected by the composition of the biomass with $12 \%$ solubilization of low S/G lignin poplar ( $\left.20 \mathrm{~g} \mathrm{~L}^{-1} \mathrm{DW}\right)$ and $20 \%$ solubilization of high S/G lignin poplar (20 g L-1 DW) [17]. The amount of solubilization of biomass by $C$. thermocellum is affected by biomass loading, with total cellulose solubilization decreasing from $76 \%$ to $58 \%$ and $63 \%$ to $37 \%$ as switchgrass loadings were increased from 9.2 to $92 \mathrm{~g} \mathrm{~L}^{-1}$ and 10 to $50 \mathrm{~g} \mathrm{~L}^{-1}$ (dry weight), respectively, although Avicel was completely consumed at all loadings [30, 31]. In the work reported here, we achieved $14 \%$ solubilization of poplar wood at $11.4 \mathrm{~g} \mathrm{~L}^{-1}$ solids loading after 5 -d of fermentation (Fig. 1 and Additional File 1: Fig. S1). The prior results and those reported here confirm that $C$. thermocellum can solubilize non-pretreated woody lignocellulosic biomass, but that additional pretreatment or alternative biomass modifications are necessary to achieve efficient conversion of biomass at high solids loadings [30, 32].

The plant biomass-solubilizing enzymes produced by $\mathrm{C}$. thermocellum are expressed and mostly arranged on multi-enzyme structures known as cellulosomes and it appears that the microbe can regulate the enzymes on the cellulosomes based on the substrate and growth conditions [24-27, 33]. $C$. thermocellum hydrolysis of cellulose in poplar biomass was reported to result in solubilization of $17 \%$ to $18 \%$ of the glucan in recovered post-fermentation solid residues compared to the controls [17]. Another 
study, using confocal microscopy, revealed a $49 \%$ reduction of cellulose signals in post-fermentation biomass and a concomitant $28 \%$ reduction in total glucose after CBP with $C$. thermocellum on $P$. deltoides [21]. In the current study, the cellulose content in post-fermented CBP solid residues was shown to decrease $21 \%$ and $25 \%$ based on Saeman hydrolysis $(\mathrm{SH})$ and methylated alditol (MA) analysis, respectively (Fig. 5). These results compare well with the prior studies [17, 21]. The limited hydrolysis by C. thermocellum of cellulose in poplar biomass results in $75 \%$ to $79 \%$ of the cellulose being retained in the poplar solid residues after $5 \mathrm{~d}$ of fermentation.

It is interesting to consider the effect of lignin quantity and composition on the ability of $C$. thermocellum CBP to solubilize cellulose in poplar biomass. The $\mathrm{CBI}$ reference poplar has a mutation in the 5-enolpyruvylshikimate-2- phosphate (EPSP) synthase gene and is among multiple low recalcitrance poplar natural variants affected in lignin biosynthesis with high $S / G$ ratios that have been positively correlated with glucan solubilization [16]. Here, we confirmed that the total lignin content of the CBI reference poplar $(23.3 \%)$ is significantly lower than that of the BESC standard poplar $(27.05 \%)$ on a biomass dry weight basis [16]. Analysis of the fate of the lignin in the post-fermentation solid residues of the $\mathrm{CBI}$ reference poplar showed that after CBP there was greater total lignin (25\%), syringyl (S) lignin (22 to $26 \%$ ), and guaiacyl (G) lignin (11 to $15 \%$ ) content and a higher S/G ratio (6 to $14 \%$ ) in the solid residues, but no major change in the $p$-hydroxyphenyl $(\mathrm{H})$ subunits compared to the fermentation control solid residues (Fig. 4a-e). These results are consistent with prior reports indicating that $\mathrm{S} / \mathrm{G}$ ratios and lignin composition impact hydrolysis of poplar wood by $C$. thermocellum and that lignin content increases in the insoluble residue generated during CBP fermentation [16, 17, 21]. The substantial increase in the S- and G-lignin in the CBP fermentation solid residues agrees with the earlier finding that lignin accessible surface increased (S - lignin increased 30\%, G - lignin increased 11\%), whereas the cellulose surface decreased (49\%) during microbial hydrolysis in poplar [21].

The hemicellulose content in poplar species is reported to range from $16 \%$ to $23 \%[22,34]$. The main non-cellulosic polysaccharide in hardwoods is the hemicellulose glucuronoxylan. Xylan constitutes from $13.4 \%$ to $22.4 \%$ of wood dry weight in poplar [22,34]. The second most abundant non-cellulosic polysaccharide in hardwoods is either glucomannan or pectin [34]. In addition to cellulases, cellulosomes from $C$. thermocellum contain xylanases, pectinases, and other accessory glycoside hydrolase enzymes $[24,25]$. For example, $C$. thermocellum expresses multiple hemicellulose degrading enzymes, including xylanases (XynA, XynC, XynD, XynE, XynY, XynZ) [35-40], xyloglucanase (XghA) [37], mannanase (Man26A, B) [41, 42], and other hemicellulases, such as ( $\beta-1,3-1,4-$ glucanase, LicB [43] and a chitinase (endochitinase, Chi18A [44]) the latter which, although not a hemicellase hydrolyzes a structure with a comparable b-1,4-linked backbone. Interestingly, all the known cellulosomal xylanases contain either the carbohydrate-binding domain 22 (CBM22) or CBM6 which bind to xylan and cellulose [38, 45], suggesting that these enzymes are specifically targeting xylan associated with cellulose. However, although $C$. thermocellum is able to break down xylan, it is unable to ferment hemicellulose solubilized from the lignocellulosic biomass $[24,46]$. Accordingly, we show that the amounts of non-cellulosic sugars attributable to hemicelluloses present in the liquor during $C$. thermocellum CBP fermentation are significantly greater than the amounts in the liquor of the fermentation controls. Specifically, the liquor 
during $C$. thermocellum CBP fermentation contained $41 \%$ to $267 \%$ more xylose $(69 \%$ to $368 \% \mathrm{mg} /$ total solubilized liquor); $35 \%$ to $80 \%$ more mannose ( $61 \%$ to $130 \% \mathrm{mg} /$ total solubilized liquor); and $19 \%$ to $35 \%$ more non-cellulosic glucose ( $42 \%$ to $72 \% \mathrm{mg} /$ total solubilized liquor) (Fig. 3 and Additional File 3: Fig. S3) per $\mathrm{mg} /$ starting biomass than the liquor of the fermentation controls over the $120 \mathrm{~h}$ incubation period. Concomitantly, there was a significant $10-18 \%$ reduction in the total non-cellulosic sugar content in the post-fermentation CBP solid residues from $24 \mathrm{~h}$ to $120 \mathrm{~h}$ (Fig. 6 and Additional File 7: Fig. S7) including a 7 to $14 \% \mathrm{mg} /$ starting material reduction in xylose, a 13 to $36 \% \mathrm{mg} /$ starting material reduction in mannose and a 13 to $23 \% \mathrm{mg} / \mathrm{starting}$ material reduction in non-cellulosic glucose. The retention of significant amounts of the solubilized Xyl, Man, and non-cellulosic Glc in the fermentation liquor supports the hypothesis that $C$. thermocellum cannot grow on pentose sugars and also is not able to ferment hemicellulosic sugars like xylose and mannose from poplar wood [24, 47]. The hemicellulose-solubilizing enzymes are suggested to function by removing hemicelluloses from the biomass in order to expose the preferred cellulose substrate to $C$. thermocellum for effective cellulosomal solubilization $[24,48]$.

Enzymatic and chemical removal of xylan is known to enhance the accessibility of glucanases to cellulose in lignocellulosic biomass [49]. However, in the current study, the total solids solubilization of biomass by CBP was only $14 \%$ greater than in the fermentation controls. It has previously been reported that xylose oligomers have inhibitory effects on the enzymatic hydrolysis, conversion rates, and hydrolysis yield of $C$. thermocellum grown on plant biomass; as well as the hydrolysis of Avicel [30, 5052]. When considered together with the reduced xylose content in the solid residues (Fig. 6 and Additional File 7: Fig. S7), the increased amounts of xylose in the $C$. thermocellum CBP liquor (Fig. 3 and Additional File 3: Fig. S3) are consistent with the hypothesis that xylose oligomers are accumulating and may be inhibiting the solubilization of poplar wood by $C$. thermocellum. These results indicate that efforts to enable $C$. thermocellum to metabolize and utilize hemicellulose may enable better solubilization of poplar biomass.

C. thermocellum expresses multiple pectin solubilizing enzymes including three endopectinlyases (PL1A, PL1B and PL9) [53] and a reported rhamnogalacturonan I (RG-I) lyase (PL11 family: RGLf [54]). RGLf has activity against homogalacturonan and RG-I preparations containing homogalacturonan. All these enzymes exist as modular enzymes that contain a carbohydrate binding module and function in cellulosomes. Interestingly, whereas PL1A contains the carbohydrate-binding domain 6 (CBM6) that binds to cellulose or hemicellulose, RGLf; as well as the multifunctional PL1B and PL9, contains CBM35 that has recently been shown to bind RG-I [55], indicating that solubilization of pectin is genetically programmed into $C$. thermocellum for biomass solubilization. The cell wall sugar composition analyses reported here show the largest reduction of GalA (56-71\%) content compared to the other non-cellulosic sugars in the CBP biomass residues compared to the fermentation controls (Fig. 6, Additional File 7: Fig. S7, Table 1). These results suggest that $C$. thermocellum efficiently utilizes polygalacturonic acid (homogalacturonan) in poplar wood as a carbon source, as previously reported for sugar beet [13]. Surprisingly; however, there was a significant increase in the amounts of rhamnose (Rha, $14 \%$ to $27 \%$ ), a major sugar in the RG-I backbone, in the solids residue after the first $24 \mathrm{~h}$ of $C$. thermocellum fermentation compared to the control fermentations, suggesting that $C$. thermocellum was 
not efficiently solubilizing RG-I. Also, there was no change in the amount of arabinose and galactose, sugars abundant in the side branches of RG-I.

Compared to the fermentation control, $C$. thermocellum hydrolysis of poplar biomass removed only $17 \%$ more of the total non-cellulosic sugars after $5 \mathrm{~d}$ of fermentation, indicating that the residual poplar wood still retained the bulk of the non-cellulosic wall polysaccharides (Additional File 7: Fig. S7B). Because fermentation of non-pretreated poplar by $C$. thermocellum resulted in $7 \%$ total solids solubilization compared to the fermentation control (Fig. 1) and there was a greater retention of glycosyl residues associated with RG-I in the post-fermentation solid residues than in the fermentation control, we hypothesize that RG-I and its side chains may be resistant to solubilization by $C$. thermocellum and potentially contribute to the limited hydrolysis of non-pretreated woody biomass by $C$. thermocellum. Our current hypothesis is supported by the recent finding that poplar wood RG-I is a key determinant of cellcell adhesion in wood [10]. Yang and coworkers showed that treatment of poplar wood with acidic chlorite and dilute alkali after digestion with the RG-I degrading enzyme RG-lyase resulted in more separation of the poplar wood into single cells than treatement with acidic chlorite and dilute alkali alone, supporting a role for RG-I in cell:cell adhesion. Cell separation in the woody biomass was also increased by reducing the RG-I content in the wood by transgenic expression of an Arabidopsis thaliana gene encoding an RG-lyase (AtRGIL6) in poplar [10], confirming a role for RG-I in wood cell wall architecture and cell:cell adhesion.

The cellulosomal cellulolytic activities and non-cellulosic xylanases and pectinolytic activities makes $C$. thermocellum a promising CBP microbe for production of fuels and chemicals from lignocellulosic biomass. The microbe by itself; however, is not able to achieve effective solubilization of all the cellulosic and non-cellulosic polysaccharides from some biomass feedstocks. The low percentage solubilization of the untreated woody biomass is a bottleneck. We previously reported that pectin contributes to biomass recalcitrance and saccharification yield although it is present in relatively low abundance in woody biomass $[4,56-58]$. Furthermore, the deletion of a pectinase gene cluster in the thermophile Caldicellulosiruptor bescii resulted in reduced growth of the mutated microbe on Arabidopsis, switchgrass, and poplar biomass, supporting a role for pectin in biomass recalcitrance [59]. The present study suggests that $C$. thermocellum is unable to effectively degrade the RGI component of pectin, making RG-I a potential target for improving the ability of $C$. thermocellum to effectively solubilize woody biomass.

\section{Materials And Methods}

\section{Populus biomass preparation}

Populus trichocarpa genotype GW-9947 was selected as the woody reference biomass for CBI (The Center for Bioenergy Innovation). GW-9947 is an elite natural variant from the BESC (BioEnergy Science Center) top line candidates with high ethanol yields, low lignin, and high S/G lignin [16]. Trees were 
harvested, debarked, chipped, air dried and milled to a particle size of 20 mesh $(0.85 \mathrm{~mm})$. The ground samples were used for the described analyses.

\section{Consolidated Bioprocessing (CBP)}

\section{C. thermocellum inoculum culture medium, growth medium and growth conditions}

Fermentations were carried out using $C$. thermocellum strain DSM1313. Cultures were grown in $1 x$ MTC (Medium for Thermophilic Clostridia) solution. The MTC stock solutions were diluted with $\mathrm{H}_{2} \mathrm{O}$ and the desired carbon source to reach $1 x$ concentration. The medium was prepared as described in Holwerda and coworkers [60] with the only exception being that $\mathrm{CBI}$ reference poplar was used as the carbon source ( $5 \mathrm{~g} / \mathrm{L}$ glucan loading) to replace Avicel or cellobiose. For seed train cultures, the same medium was used except that $5 \mathrm{~g} / \mathrm{L}$ cellobiose was the primary carbon source for initial serum bottle seeds and 5 $\mathrm{g} / \mathrm{L}$ Avicel was the primary carbon source for the seed bioreactors. All growth medium was prepared at $\mathrm{pH} 7.0$ and maintained at this level for all bioreactor experiments.

For bioreactor preparation, a 2x MTC solution was prepared and filter sterilized without the carbon source. This solution was placed in an anaerobic chamber under $85 \% \mathrm{~N}_{2}, 10 \% \mathrm{CO}_{2}$, and $5 \% \mathrm{H}_{2}$ until the bioreactors for carrying out the fermentations were sterilized. This $2 x$ stock was then aseptically added to the autoclaved reactors already containing water and $\mathrm{CBI}$ reference poplar under a sterilized biological safety cabinet to bring the total volume to a $1 \times$ MTC concentration.

Serum bottles were prepared by making a $1 \mathrm{x}$ MTC stock that included cellobiose and allowing the solution to reach anaerobic conditions in the anaerobic chamber described above. The medium was then transferred to the desired serum bottle size inside the chamber, capped, and stored at $4^{\circ} \mathrm{C}$ until ready for use. Serum bottle seeds were allowed to proliferate to an $\mathrm{OD}_{600}$ of approximately 0.6 before transferring at $10 \% \mathrm{v} / \mathrm{v}$ to the $500 \mathrm{~mL}$ Avicel seed reactors. Avicel seed reactors were grown for approximately $24 \mathrm{~h}$ before transferring at $10 \% \mathrm{v} / \mathrm{v}$ to the $\mathrm{CBI}$ poplar containing reactors. Avicel seed cultures were monitored for ideal transferring phase by following total base addition $(2 \mathrm{M} \mathrm{KOH})$ and visual confirmation of a healthy culture under a microscope.

\section{Fermentations}

Six Sartorius vessels of $0.5 \mathrm{~L}$ total working volume were set up identically for fermentation. A total of 5.68 grams of CBI poplar (80/20 mesh sieved) was loaded into each reactor to reach a glucan loading of $5 \mathrm{~g} / \mathrm{L}(0.5 \% \mathrm{w} / \mathrm{v})$. The reactor, biomass, and water were autoclaved for $30 \mathrm{~min}$ at $121^{\circ} \mathrm{C}$. MTC media components were then added post-autoclaving as described above. Cultures were controlled at $\mathrm{pH} 7.0$ with $2 \mathrm{~N} \mathrm{HCl}$ and $2 \mathrm{~N} \mathrm{KOH}$ as the acid and base, respectively. Temperature was maintained at $60^{\circ} \mathrm{C}$, agitation at $150 \mathrm{rpm}$ minimum to keep biomass from accumulating on the bottom of the reactor, and 50 $\mathrm{ccm}$ of $\mathrm{N}_{2}$ was sparged through the reactor headspace to maintain anaerobic conditions. All reactors were inoculated with a $10 \%(\mathrm{v} / \mathrm{v})$ inoculum of DSM1313 C. thermocellum from a common seed culture containing $5 \mathrm{~g} / \mathrm{L}$ Avicel. At 24, 48, and $120 \mathrm{~h}$, two reactors each were shut down and harvested 
(Additional File 1: Fig. S1). Similar reactors were set up to act as controls that were not inoculated with $C$. thermocellum and instead brought up to the final volume with medium.

\section{Separations and quantification of total biomass solubilization}

To separate the undigested solids from the liquor, the reactor contents were transferred to centrifuge bottles and spun at 10,000 RPM for 15 min. The supernatant was removed by pipetting, frozen for storage, and this fraction was referred to as 'liquor'. The solids residue was washed with distilled water and centrifuged again under the same conditions. The wash fraction was removed by pipetting and the residual solids were dried for $5 \mathrm{~d}$ at $60^{\circ} \mathrm{C}$. The dried biomass was weighed, and the total solids solubilization was calculated by dividing the total recovered biomass after fermentation by the initial biomass loaded into the reactors at the start of fermentation.

\section{Cell wall analysis}

Alcohol insoluble residue (AIR) from solid residues post fermentation was prepared as previously described $[4,15]$. Prior to the analysis, AIR samples were treated with alpha-amylase $(0.47 \mathrm{U}$ per $\mathrm{mg}$ biomass, Sigma Cat \# A6255) in $100 \mathrm{mM}$ ammonium formate $(\mathrm{pH} 6.0)$ at $25^{\circ} \mathrm{C}$ for $48 \mathrm{~h}$ to remove starch, followed by three $\mathrm{ddH}_{2} \mathrm{O}$ and two acetone washes. The AIR samples were then kept in fume hood for 72 $\mathrm{h}$ to dry. Glycosyl residue composition analysis of the AIR ( 2 mg) and liquor (100 to $300 \mu \mathrm{g})$ was by GCMS of trimethylsilyl (TMS) derivatization of the monosaccharide methyl glycosides produced from the sample by acidic methanolysis as previously described $[15,57,61]$. Briefly, the samples were aliquoted into individual tubes and inositol $(20 \mu \mathrm{g})$ was added as an internal standard. The samples were hydrolyzed for $18 \mathrm{~h}$ at $80^{\circ} \mathrm{C}$ in $1 \mathrm{M}$ methanolic- $\mathrm{HCl}$ and the walls were then derivatized with $200 \mu \mathrm{L}$ of TriSil and heated to $80^{\circ} \mathrm{C}$ for $20 \mathrm{~min}$. After filtering through packed glass wool, the dried samples were resuspended in $150 \mu \mathrm{L}$ hexane and $1 \mu \mathrm{L}$ of sample was injected into the GC-MS as described earlier [15].

\section{Saeman hydrolysis method of cellulose quantification}

One method to determine cellulose content was to first quantify the total glucose content of the sample by Saeman hydrolysis of the cellulose in the sample $[19,20]$ followed by glycosyl residue composition analysis to give the total glucose (Glc) content and then subtracting from that the non-cellulosic glucose content as determined by TMS GC-MS (described above). The Saeman hydrolysis method hydrolyzes crystalline cellulose. Specifically, around $2 \mathrm{mg}$ of AIR solid residue with $20 \mathrm{mg}$ of inositol internal standard was hydrolyzed in $72 \% \mathrm{H}_{2} \mathrm{SO}_{4}$ for $1 \mathrm{~h}$ with vortexing every 15 minutes at room temperature. Samples were diluted to $1 \mathrm{M} \mathrm{H}_{2} \mathrm{SO}_{4}$ and heated to $121^{\circ} \mathrm{C}$ for $1 \mathrm{~h}$. Cooled samples were neutralized with $0.25 \mathrm{M} \mathrm{Ba}(\mathrm{OH})_{2}$. Supernatants were collected after centrifugation at $3600 \mathrm{xg}$ for $20 \mathrm{~min}$ and dried down by lyophilization. The quantification of glucose in the Saeman hydrolysis sample was by the GC-MS of TMS-derivatized methyl glycosides as described above [15]. This value represented the total cellulosic plus non-cellulosic Glc in the sample. The amount of fibrillary cellulose was obtained by subtracting the 
amount of non-cellulosic Glc obtained from a separate TMS GC-MS composition analysis from the total Glc value to give the total cellulose content of the sample.

\section{Methyl alditol (MA) glycosyl residue composition method for cellulose quantification}

Glycosyl residue composition analysis was performed by GC-MS of the per- $O$-methyl alditol (MA) derivatives of the monosaccharides produced from the sample as described previously by Black and coworkers [23]. Permethylation of the AIR samples $(\sim 100 \mu \mathrm{g})$ was by two rounds of treatment with sodium hydroxide (15 min) and methyl iodide (45 min). After exraction in DCM, the samples were hydrolyzed using $2 \mathrm{M} \mathrm{TFA}\left(2 \mathrm{~h}\right.$ in sealed tube at $121^{\circ} \mathrm{C}$ ), reduced with $\mathrm{NaBD}_{4}$, and re-permethylated as before. The resulting MAs were analyzed on an Agilent 7890A GC interfaced to a 5975C MSD (mass selective detector, electron impact ionization mode); separation was performed on a $30 \mathrm{~m}$ Supelco Equity1 bonded phase fused silica capillary column. The value of Glc obtained from the MA method represented the total Glc content of the sample. The Glc attributed to cellulose was calculated by subtracting the non-cellulosic Glc content as determined by TMS GC-MS (described above) from the total Glc content to give the cellulose content.

\section{Lignin}

The Complex Carbohydrate Research Center (CCRC) high-throughput pyrolysis molecular beam mass spectrometry (MBMS) method [62] was used to quantify the lignin content and S/G lignin monomer ratio of the solid residues post-fermentaion that remained after CBP including the fermentation control. The samples were prepared in duplicate by weighing 1.5 to $3.0 \mathrm{mg}$ into a stainless metal cup, which was single-shot pyrolyzed (Frontier Lab) at $500^{\circ} \mathrm{C}$ to produce volatile compounds. The volatile compounds were analyzed for lignin using a molecular beam mass spectrometer (Extrel Core Mass Spectrometers). The raw data were processed through UnscramblerX 10.1 software to obtain the principal components and raw lignin data. NIST 8492 (26.2\% lignin content) and Aspen standards were also pyrolyzed and analyzed in the same manner and in the same batch as the unknown samples. Both standards were used for data quality control. Additionally, NIST 8492 was used to correct the raw lignin data.

\section{Abbreviations}

AIR:Alcohol-insoluble residue

Ara: arabinose

CBP. Consolidated bioprocessing

Gal: galactose

GalA: galacturonic acid

GC-MS: gas chromatography-mass spectrometry 
Glc: glucose

Man:mannose

MA: Methylated Alditol

MBMS:Molecular beam mass spectrometer

Rha: rhamnose

RG-I: Rhamnogalacturonan I

TMS:Trimethylsilyl

Xyl:xylose

\section{Declarations}

\section{Ethics approval and consent to participate}

Not applicable.

\section{Consent for publication}

All the authors agree to the publication of this manuscript.

\section{Competing interests}

The authors declare that they have no competing interests.

\section{Funding}

Funding provided by The Center for Bioenergy Innovation a U.S. Department of Energy Research Center supported by the Office of Biological and Environmental Research in the DOE Office of Science. Some of the carbohydrate analyses were also partially supported by the U.S. Department of Energy, Office of Science, Basic Energy Sciences, Chemical Sciences, Geosciences and Biosciences Division, under award \#DE-SC0015662. This work was authored in part by Alliance for Sustainable Energy, LLC, the manager and operator of the National Renewable Energy Laboratory for the U.S. Department of Energy (DOE) under Contract No. DE-AC36-08G028308. The views expressed in the article do not necessarily represent the views of the DOE or the U.S. Government. The U.S. Government retains and the publisher, by accepting the article for publication, acknowledges that the U.S. Government retains a nonexclusive, paidup, irrevocable, worldwide license to publish or reproduce the published form of this work, or allow others to do so, for U.S. Government purposes.

\section{Authors' contributions}


$A K B, Y J B$, and DM planned and designed the research. AKB, NNH, IMB, MAA, SSM, DR, PA and MH performed and evaluated the research. $\mathrm{AKB}, \mathrm{NNH}, \mathrm{YJB}$, and $\mathrm{DM}$ analyzed data. AKB, NH, YJB, MEH, and DM wrote the manuscript. All authors read and approved the final manuscript.

\section{Acknowledgements}

We thank CCRC Analytical Services for lignin analysis. We also thank Sheilah Dixon Huckabee for administrative assistance, and Gene Osuoha for assistance with the sample processing.

\section{Availability of data and material}

All data generated or analyzed during this study are included in the article/supplementary material.

\section{References}

1. Lynd LR, Wyman CE, Gerngross TU. Biocommodity Engineering Biotechnol Prog. 1999;15(5):777-93.

2. Corona $\mathrm{B}$, et al., Towards sustainable development through the circular economy-A review and critical assessment on current circularity metrics. Resources, Conservation and Recycling, 2019. 151: p. 104498.

3. Akinosho $\mathrm{H}$, et al. The emergence of Clostridium thermocellum as a high utility candidate for consolidated bioprocessing applications. Frontiers in chemistry. 2014;2:66-6.

4. Biswal AK, et al. Sugar release and growth of biofuel crops are improved by downregulation of pectin biosynthesis. Nat Biotechnol. 2018;36(3):249-57.

5. Tan L, et al. An Arabidopsis Cell Wall Proteoglycan Consists of Pectin and Arabinoxylan Covalently Linked to an Arabinogalactan Protein. Plant Cell. 2013;25(1):270.

6. Himmel ME, et al. Biomass Recalcitrance: Engineering Plants and Enzymes for Biofuels Production. Science. 2007;315(5813):804-7.

7. Somerville C, et al. Feedstocks for Lignocellulosic Biofuels. 2010;329(5993):790-2.

8. Caffall $\mathrm{KH}$, Mohnen D. The structure, function, and biosynthesis of plant cell wall pectic polysaccharides. Carbohydr Res. 2009;344(14):1879-900.

9. Šola K, et al. RUBY, a Putative Galactose Oxidase, Influences Pectin Properties and Promotes Cell-ToCell Adhesion in the Seed Coat Epidermis of Arabidopsis. Plant Cell. 2019;31(4):809.

10. Yang $\mathrm{H}$, et al. Rhamnogalacturonan-l is a determinant of cell-cell adhesion in poplar wood. Plant Biotechnol J. 2020;18(4):1027-40.

11. Mazzoli R, Olson DG. Chapter Three - Clostridium thermocellum: A microbial platform for high-value chemical production from lignocellulose, Advances in Applied Microbiology, G.M. Gadd and S. Sariaslani, Editors. 2020, Academic Press. pp. 111-61.

12. Aburaya $\mathrm{S}$, et al. Elucidation of the recognition mechanisms for hemicellulose and pectin in Clostridium cellulovorans using intracellular quantitative proteome analysis. AMB Express. 2015;5(1):29. 
13. Spinnler HE, Lavigne B, Blachere H. Pectinolytic activity of Clostridium thermocellum: Its use for anaerobic fermentation of sugar beet pulp. Appl Microbiol Biotechnol. 1986;23(6):434-7.

14. Holwerda EK, et al. Multiple levers for overcoming the recalcitrance of lignocellulosic biomass. Biotechnol Biofuels. 2019;12(1):15.

15. Biswal AK, et al. Comparison of four glycosyl residue composition methods for effectiveness in detecting sugars from cell walls of dicot and grass tissues. Biotechnol Biofuels. 2017;10:182.

16. Akinosho H, et al. Lignin Exhibits Recalcitrance-Associated Features Following the Consolidated Bioprocessing of Populus trichocarpa Natural Variants. ChemistrySelect. 2017;2(33):10642-7.

17. Dumitrache A, et al. Consolidated bioprocessing of Populus using Clostridium (Ruminiclostridium) thermocellum: a case study on the impact of lignin composition and structure. Biotechnol Biofuels. 2016;9(1):31.

18. Akinosho HO, et al. Elucidating the Structural Changes to Populus Lignin during Consolidated Bioprocessing with Clostridium thermocellum. ACS Sustainable Chemistry Engineering. 2017;5(9):7486-91.

19. Persson S, et al. The Arabidopsis irregular xylem8 Mutant Is Deficient in Glucuronoxylan and Homogalacturonan, Which Are Essential for Secondary Cell Wall Integrity. Plant Cell. 2007;19(1):237.

20. Saeman JF, Bubl JL, Harris EE. Quantitative Saccharification of Wood and Cellulose. Industrial \& Engineering Chemistry Analytical Edition, 1945. 17(1): 35-7.

21. Dumitrache $A$, et al. Cellulose and lignin colocalization at the plant cell wall surface limits microbial hydrolysis of Populus biomass. Green Chem. 2017;19(9):2275-85.

22. Sannigrahi P, Ragauskas AJ, Tuskan GA. Poplar as a feedstock for biofuels: A review of compositional characteristics. Biofuels Bioprod Biorefin. 2010;4(2):209-26.

23. Black I, Heiss C, Azadi P. Comprehensive Monosaccharide Composition Analysis of Insoluble Polysaccharides by Permethylation To Produce Methyl Alditol Derivatives for Gas Chromatography/Mass Spectrometry. Anal Chem. 2019;91(21):13787-93.

24. Raman B, et al. Impact of Pretreated Switchgrass and Biomass Carbohydrates on Clostridium thermocellum ATCC 27405 Cellulosome Composition: A Quantitative Proteomic Analysis. PLOS ONE. 2009;4(4):e5271.

25. Leis B, et al. Comparative characterization of all cellulosomal cellulases from Clostridium thermocellum reveals high diversity in endoglucanase product formation essential for complex activity. Biotechnol Biofuels. 2017;10(1):240.

26. Bayer EA, et al. From cellulosomes to cellulosomics. The Chemical Record. 2008;8(6):364-77.

27. Xu Q, et al. Dramatic performance of Clostridium thermocellum explained by its wide range of cellulase modalities. Science Advances. 2016;2(2):e1501254.

28. Ellis LD, et al. Closing the carbon balance for fermentation by Clostridium thermocellum (ATCC 27405). Biores Technol. 2012;103(1):293-9. 
29. Holwerda EK, et al. The exometabolome of Clostridium thermocellum reveals overflow metabolism at high cellulose loading. Biotechnol Biofuels. 2014;7(1):155.

30. Shao X, Murphy SJ, Lynd LR. Characterization of reduced carbohydrate solubilization during Clostridium thermocellum fermentation with high switchgrass concentrations. Biomass Bioenerg. 2020;139:105623.

31. Verbeke TJ, Garcia GM, Elkins JG. The effect of switchgrass loadings on feedstock solubilization and biofuel production by Clostridium thermocellum. Biotechnol Biofuels. 2017;10(1):233.

32. Kothari $\mathrm{N}$, et al. Biomass augmentation through thermochemical pretreatments greatly enhances digestion of switchgrass by Clostridium thermocellum. Biotechnol Biofuels. 2018;11(1):219.

33. Zverlov VV, Kellermann J, Schwarz WH. Functional subgenomics of Clostridium thermocellum cellulosomal genes: identification of the major catalytic components in the extracellular complex and detection of three new enzymes. Proteomics. 2005;5(14):3646-53.

34. Willför S, et al. Polysaccharides in some industrially important hardwood species. Wood Sci Technol. 2005;39(8):601-17.

35. Hayashi $\mathrm{H}$, et al. Sequence of $x y n C$ and properties of $\mathrm{XynC}$, a major component of the Clostridium thermocellum cellulosome. J Bacteriol. 1997;179(13):4246-53.

36. Hayashi $\mathrm{H}$, et al. Nucleotide sequences of two contiguous and highly homologous xylanase genes xynA and xynB and characterization of XynA from Clostridium thermocellum. Appl Microbiol Biotechnol. 1999;51(3):348-57.

37. Zverlov VV, et al., Two new major subunits in the cellulosome of Clostridium thermocellum: xyloglucanase Xgh74A and endoxylanase Xyn10D. 2005. 151(10): p. 3395-3401.

38. Heinze $S$, et al. Identification of endoxylanase XynE from Clostridium thermocellum as the first xylanase of glycoside hydrolase family GH141. Sci Rep. 2017;7(1):11178.

39. Grépinet O, Chebrou MC, Béguin P. Purification of Clostridium thermocellum xylanase Z expressed in Escherichia coli and identification of the corresponding product in the culture medium of $\mathrm{C}$. thermocellum. J Bacteriol. 1988;170(10):4576-81.

40. Blum DL, et al. Feruloyl Esterase Activity of the Clostridium thermocellum Cellulosome Can Be Attributed to Previously Unknown Domains of XynY and XynZ. J Bacteriol. 2000;182(5):1346.

41. Halstead JR, et al., A family 26 mannanase produced by Clostridium thermocellum as a component of the cellulosome contains a domain which is conserved in mannanases from anaerobic fungiThe GenBank/EMBL accession number for the sequence reported in this paper is AJ242666. 1999. 145(11): p. 3101-3108.

42. Kurokawa J, et al., Sequence of the Clostridium thermocellum Mannanase Gene man26B and Characterization of the Translated Product. Bioscience, Biotechnology, and Biochemistry, 2001. 65(3): p. 548-554.

43. Luo Z, et al. Cloning of LicB from Clostridium thermocellum and its efficient secretive expression of thermostable $\beta-1,3-1,4$-glucanase. Appl Biochem Biotechnol. 2014;173(2):562-70. 
44. Zverlov VV, Fuchs K-P, Schwarz WH. Chi18A, the endochitinase in the cellulosome of the thermophilic, cellulolytic bacterium Clostridium thermocellum. Appl Environ Microbiol. 2002;68(6):3176-9.

45. Boraston $A B$, et al. Carbohydrate-binding modules: fine-tuning polysaccharide recognition. Biochem J. 2004;382(Pt 3):769-81.

46. Beri D, et al., Development of a thermophilic coculture for corn fiber conversion to ethanol. Nature Communications, 2020. 11(1): p. 1937.

47. Lynd LR, et al. Microbial Cellulose Utilization: Fundamentals and Biotechnology. Microbiol Mol Biol Rev. 2002;66(3):506.

48. Lynd LR, et al., Advances in Consolidated Bioprocessing Using Clostridium thermocellum and Thermoanaerobacter saccharolyticum, in Industrial Biotechnology. 2017. p. 365-394.

49. Cheng F, et al. Novel xylanase from a holstein cattle rumen metagenomic library and its application in xylooligosaccharide and ferulic Acid production from wheat straw. J Agric Food Chem. 2012;60(51):12516-24.

50. Kumar R, Wyman CE. Effect of xylanase supplementation of cellulase on digestion of corn stover solids prepared by leading pretreatment technologies. Bioresour Technol. 2009;100(18):4203-13.

51. Qing Q, Yang B, Wyman CE. Xylooligomers are strong inhibitors of cellulose hydrolysis by enzymes. Bioresour Technol. 2010;101(24):9624-30.

52. Zhang J, Tang M, Viikari L. Xylans inhibit enzymatic hydrolysis of lignocellulosic materials by cellulases. Bioresour Technol. 2012;121:8-12.

53. Chakraborty S, et al. Role of pectinolytic enzymes identified in Clostridium thermocellum cellulosome. PloS one. 2015;10(2):e0116787-7.

54. Dhillon A, et al. A New Member of Family 11 Polysaccharide Lyase, Rhamnogalacturonan Lyase (CtRGLf) from Clostridium thermocellum. Mol Biotechnol. 2016;58(4):232-40.

55. Dhillon A, et al. The multi-ligand binding first family 35 Carbohydrate Binding Module (CBM35) of Clostridium thermocellum targets rhamnogalacturonan I. Arch Biochem Biophys. 2018;654:194-208.

56. Biswal AK, et al. Working towards recalcitrance mechanisms: increased xylan and homogalacturonan production by overexpression of GAlactUronosylTransferase12 (GAUT12) causes increased recalcitrance and decreased growth in Populus. Biotechnol Biofuels. 2018;11:9.

57. Biswal AK, et al. Downregulation of GAUT12 in Populus deltoides by RNA silencing results in reduced recalcitrance, increased growth and reduced xylan and pectin in a woody biofuel feedstock. Biotechnol Biofuels. 2015;8(1):41.

58. Biswal AK, et al. Aspen pectate lyase PtxtPL1-27 mobilizes matrix polysaccharides from woody tissues and improves saccharification yield. Biotechnol Biofuels. 2014;7(1):11.

59. Chung $D$, et al. Deletion of a gene cluster encoding pectin degrading enzymes in Caldicellulosiruptor bescii reveals an important role for pectin in plant biomass recalcitrance. Biotechnol Biofuels. 2014;7(1):147. 
60. Holwerda EK, Hirst KD, Lynd LR. A defined growth medium with very low background carbon for culturing Clostridium thermocellum. Journal of Industrial Microbiology Biotechnology. 2012;39(6):943-7.

61. York WS, et al. Isolation and characterization of plant cell walls and cell wall components. In: Methods in Enzymology. Academic Press; 1986. pp. 3-40.

62. Hao Z, et al. Loss of Arabidopsis GAUT12/IRX8 causes anther indehiscence and leads to reduced G lignin associated with altered matrix polysaccharide deposition. Frontiers in plant science. 2014;5:357-7.

\section{Tables}

Table 1: Mass of lignin, cellulosic and non-cellulosic sugars in starting biomass and residual solids during $\mathrm{CBP}$ by $\mathrm{C}$. thermocellum. All data in $\mathrm{mg}$ per fermentation reaction, rounded to nearest integer. Zero and negative values are in bold. Gross - total solubilization of residual pellet in CPB fermenter. Net solubilization of CPB fermenter residual pellet minus fermentation control residual pellet. SH, Saeman hydrolysis; MA, Methylated alditol. 


\begin{tabular}{|c|c|c|c|c|c|c|c|}
\hline \multirow[t]{2}{*}{$\begin{array}{l}\text { Type of } \\
\text { Sugar }\end{array}$} & \multirow[t]{2}{*}{$\begin{array}{l}\text { Starting } \\
\text { Biomass } \\
(\mathrm{mg})\end{array}$} & \multicolumn{2}{|c|}{$\begin{array}{l}\text { Final Solid } \\
\text { Residue (mg) }\end{array}$} & \multicolumn{2}{|c|}{$\begin{array}{l}\text { Amount Solubilized by } \\
\text { C. } \\
\text { thermocellum CBP (mg) }\end{array}$} & \multicolumn{2}{|c|}{$\begin{array}{l}\text { Percentage (\%) } \\
\text { solubilization by C. } \\
\text { thermocellum CBP }\end{array}$} \\
\hline & & Control & CBP & Gross & Net & Gross & Net \\
\hline \multicolumn{8}{|c|}{ Cellulosic Sugar } \\
\hline $\begin{array}{l}\text { Glc } \\
\text { (SH) }\end{array}$ & 2307 & 2191 & 1722 & 585 & 469 & 25 & 21 \\
\hline $\begin{array}{l}\text { Glc } \\
\text { (MA) }\end{array}$ & 2528 & 2394 & 1789 & 739 & 605 & 29 & 25 \\
\hline \multicolumn{8}{|c|}{ Non-cellulosic sugar } \\
\hline Ara & 54 & 49 & 42 & 12 & 7 & 23 & 15 \\
\hline Rha & 25 & 20 & 25 & 0 & -5 & 0 & -27 \\
\hline Fuc & 5 & 4 & 3 & 2 & 1 & 32 & 15 \\
\hline Xyl & 1249 & 1160 & 995 & 253 & 165 & 20 & 14 \\
\hline GlcA & 13 & 12 & 12 & 1 & 0 & 11 & 3 \\
\hline GalA & 107 & 83 & 24 & 82 & 58 & 77 & 71 \\
\hline Man & 65 & 61 & 39 & 26 & 22 & 40 & 36 \\
\hline Gal & 53 & 49 & 42 & 11 & 7 & 21 & 13 \\
\hline Glc & 158 & 148 & 114 & 44 & 34 & 28 & 23 \\
\hline Total & 1729 & 1584 & 1296 & 433 & 288 & 25 & 18 \\
\hline \multicolumn{8}{|l|}{ Lignin } \\
\hline S lignin & 733 & 525 & 653 & 80 & -128 & 11 & -24 \\
\hline G lignin & 321 & 267 & 298 & 23 & -31 & 7 & -12 \\
\hline H lignin & 13 & 13 & 14 & -1 & -1 & -8 & -8 \\
\hline $\begin{array}{l}\text { Total } \\
\text { lignin }\end{array}$ & 1323 & 1017 & 1281 & 42 & -264 & 3.2 & -26 \\
\hline
\end{tabular}

Figures 


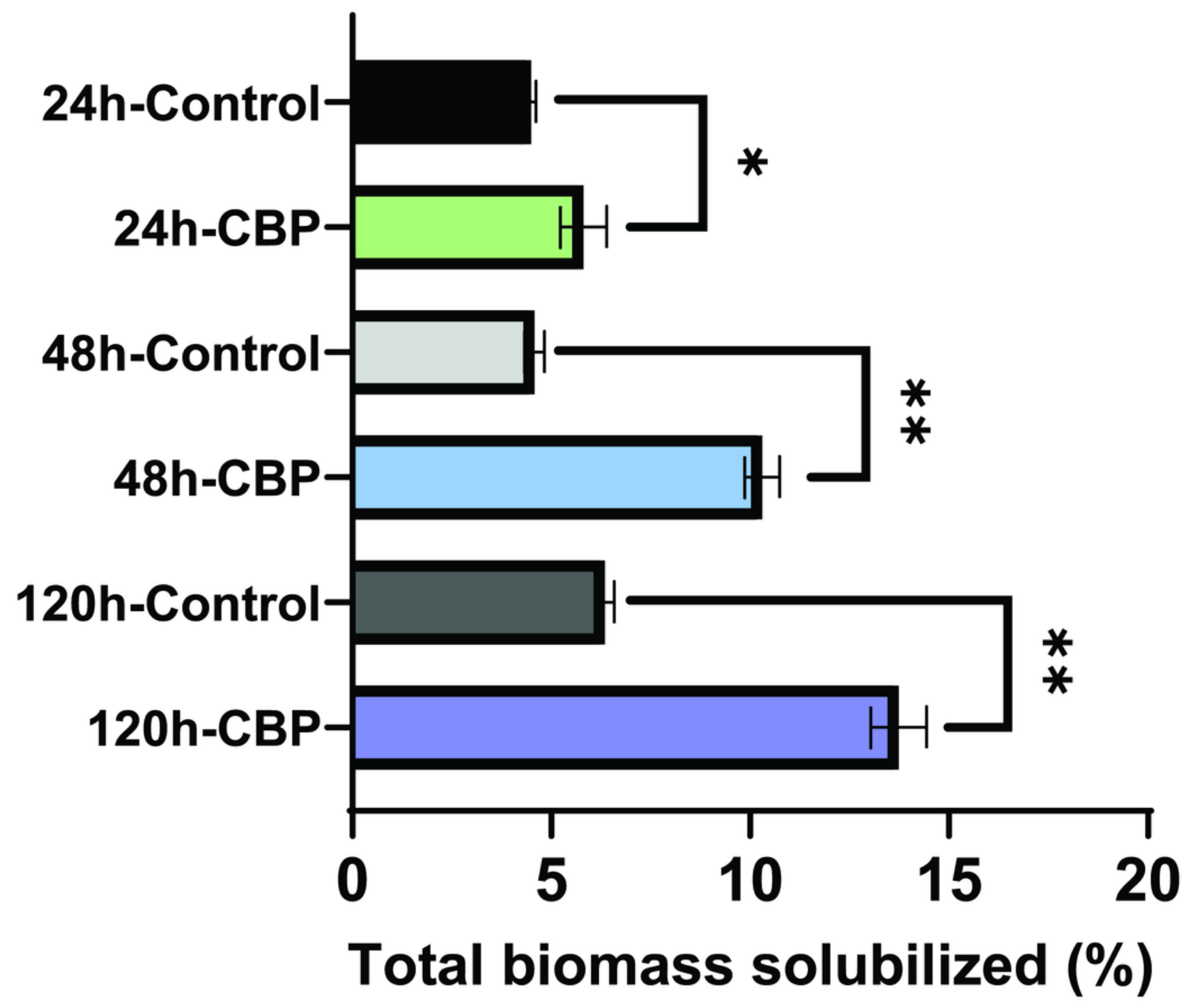

Figure 1

Total solids solubilization of CBI reference poplar biomass by C. thermocellum DSM1313 over $5 \mathrm{~d}$ at $60^{\circ} \mathrm{C}$. Poplar biomass was loaded at 5.68 grams per $500 \mathrm{~mL}$ reaction volume at the start of fermentation to achieve a $5.0 \mathrm{~g} / \mathrm{L}$ glucan loading at $0.5 \mathrm{~L}$ bioreactor scale. Uninoculated controls were included to account for any non-biological solubilization. The percentage of total biomass solubilization was calculated from the data presented in Additional File 2: Fig. S2. Data are average of two technical replicates from each of two biological replicates (i.e. two separate fermentations) \pm standard deviation, $n$ $=4$. Statistical analysis was by one-way ANOVA followed by Fisher's least significant difference method; $* \mathrm{P}<0.05, * * \mathrm{P}<0.01$. 


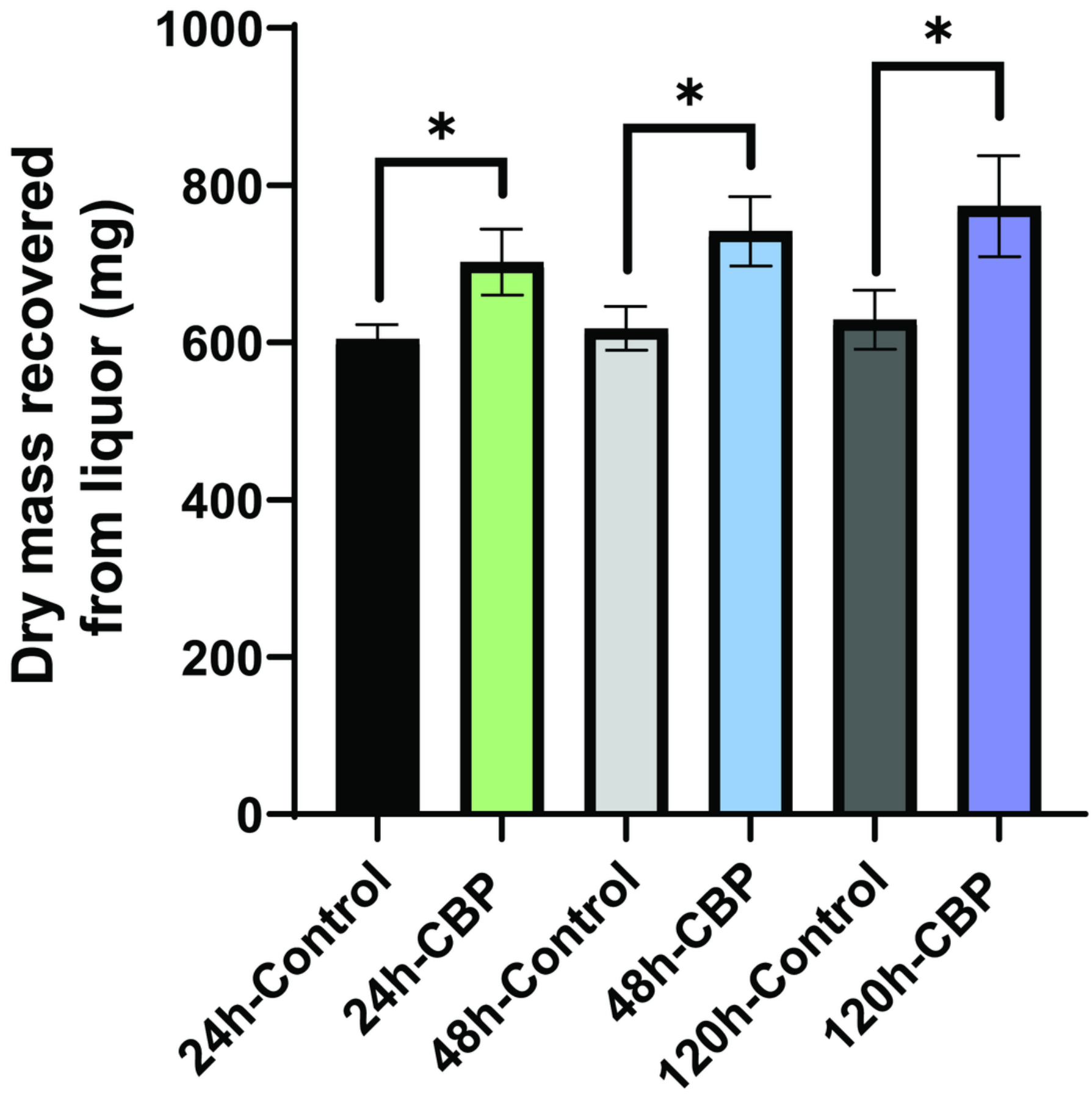

Figure 2

Total dry mass recovered from the fermentation liquor after solubilization of milled un-pretreated CBI reference poplar biomass by $\mathrm{C}$. thermocellum DSM 1313 over $5 \mathrm{~d}$ at $60^{\circ} \mathrm{C}$. Dry mass recovered from uninoculated controls was included to account for any non-biological solubilization. Liquor dry mass was collected post fermentation as described in Additional File 1: Fig. S1. Data are average from two technical replicates of each of two biological replicates (i.e. two fermentations) \pm standard deviation (SD), $n=4$. 
Statistical analysis was with one-way ANOVA followed by Fisher's least significant difference method; *P $<0.05$.

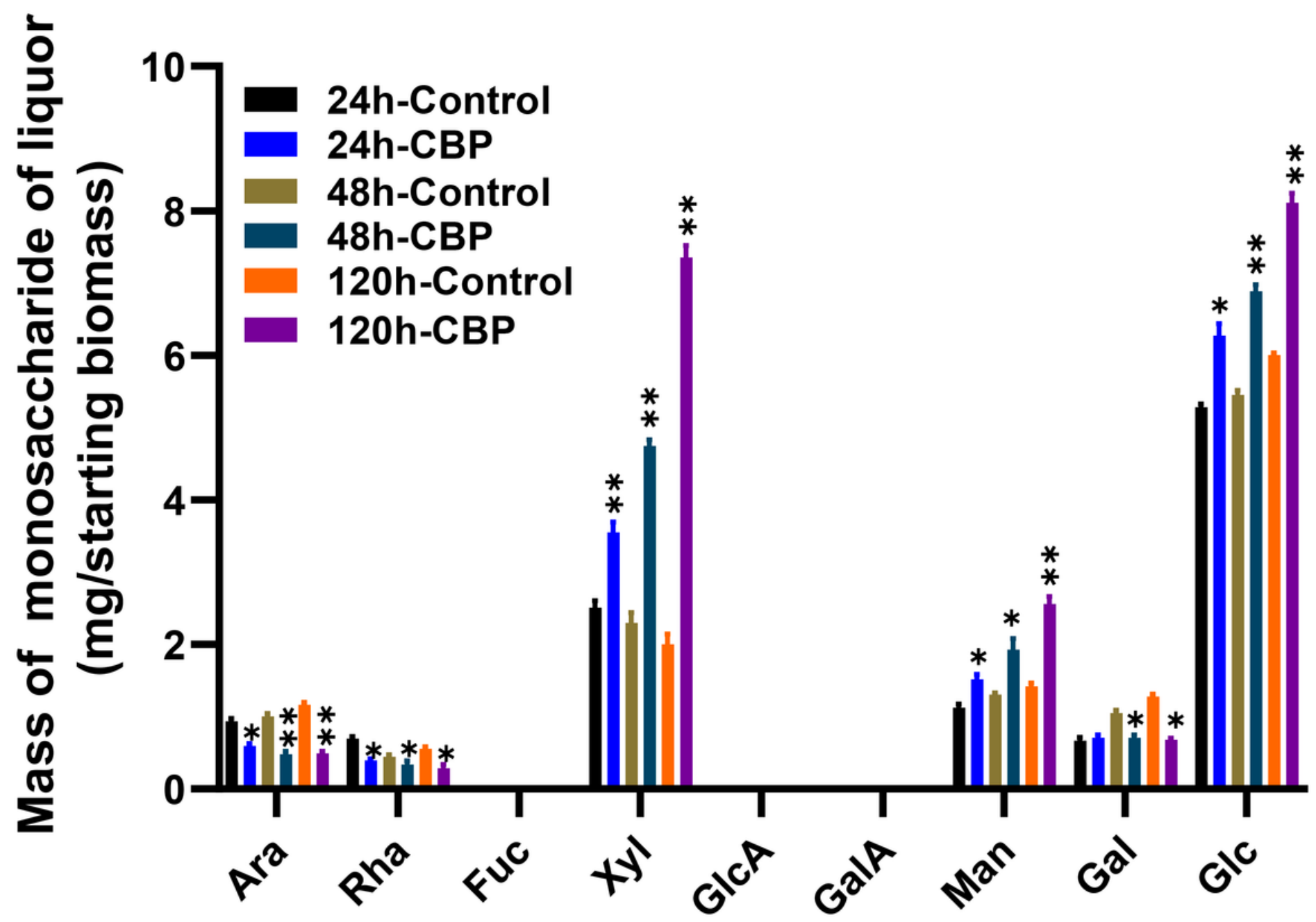

Figure 3

Glycosyl residue composition of post fermentation liquor as described in Fig. 2 by trimethylsilyl (TMS) derivatization and GC-MS. The amount of sugar is represented as average $\mathrm{mg}$ from $5.68 \mathrm{~g}$ starting materials. Mean $\pm S D$ of two biological (i.e. two fermentations) and two technical replicates $(n=4)$. $P$ values are ${ }^{*} \leq 0.05,{ }^{*} P \leq 0.001$ at significant level (one-way ANOVA followed by Fisher's least significant difference method). 

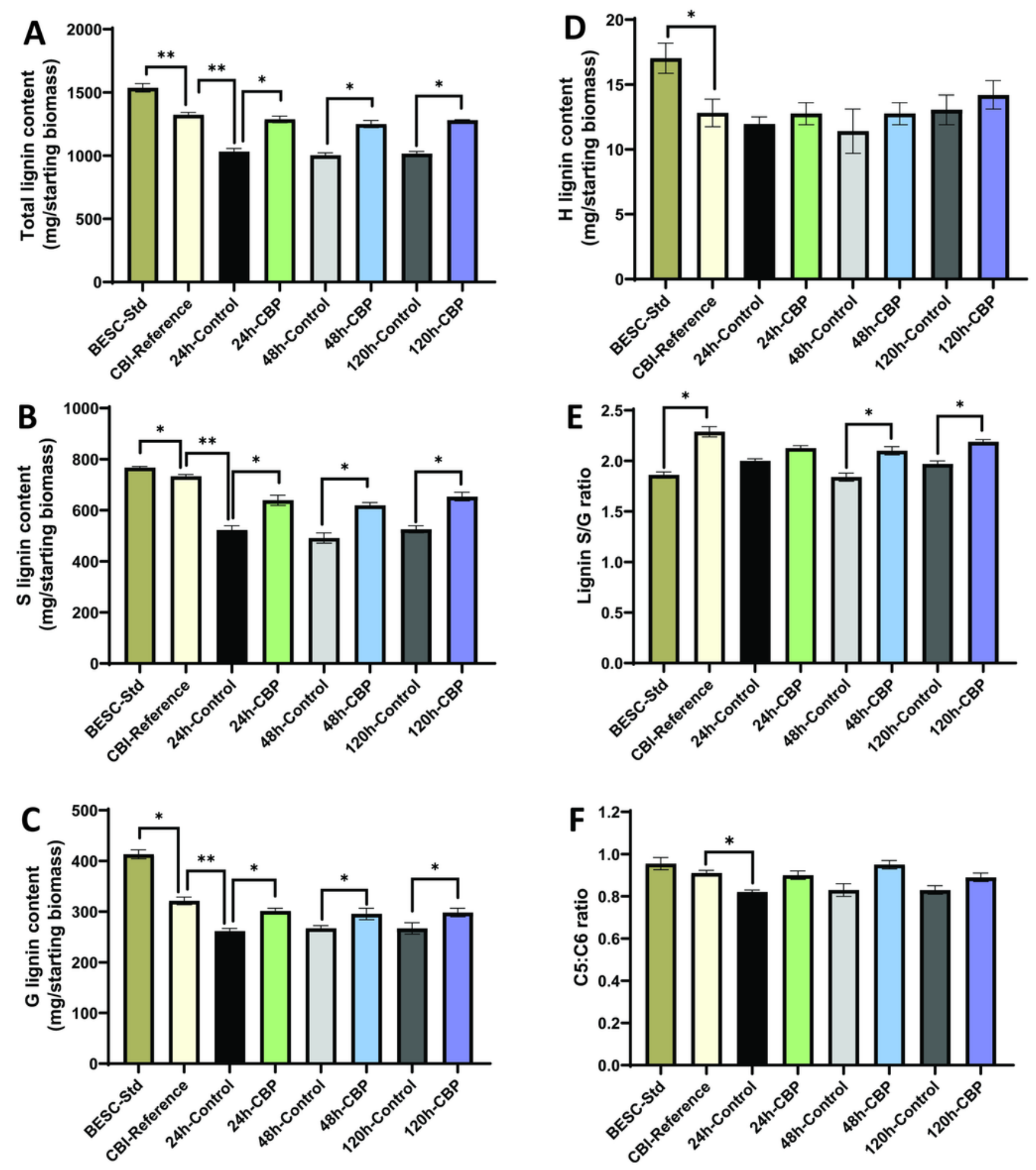

Figure 4

Lignin content and $\mathrm{S} / \mathrm{G}$ ratio of solid residue recovered after $\mathrm{C}$. thermocellum $\mathrm{CBP}$ bioconversion. (A) Total lignin, (B) S lignin, (C) G lignin, (D) H lignin content, and (E) S/G ratio and (F) C5:C6 ratio of solid residue recovered from poplar biomass after CBP bioconversion with $\mathrm{C}$. thermocellum over $120 \mathrm{~h}$ compared to fermentation controls. Total lignin, $\mathrm{S}, \mathrm{G}$ and $\mathrm{H}$ lignin contents are presented as $\mathrm{mg}$ per 
starting biomass. Statistical analysis was by one-way analysis of variance (ANOVA) followed by Fisher's method. Mean $\pm S D$, Significant $P$ values are expressed as $* P<0.05,{ }^{*} P<0.01$.

\section{A Cellulose content - Saeman hydrolysis}
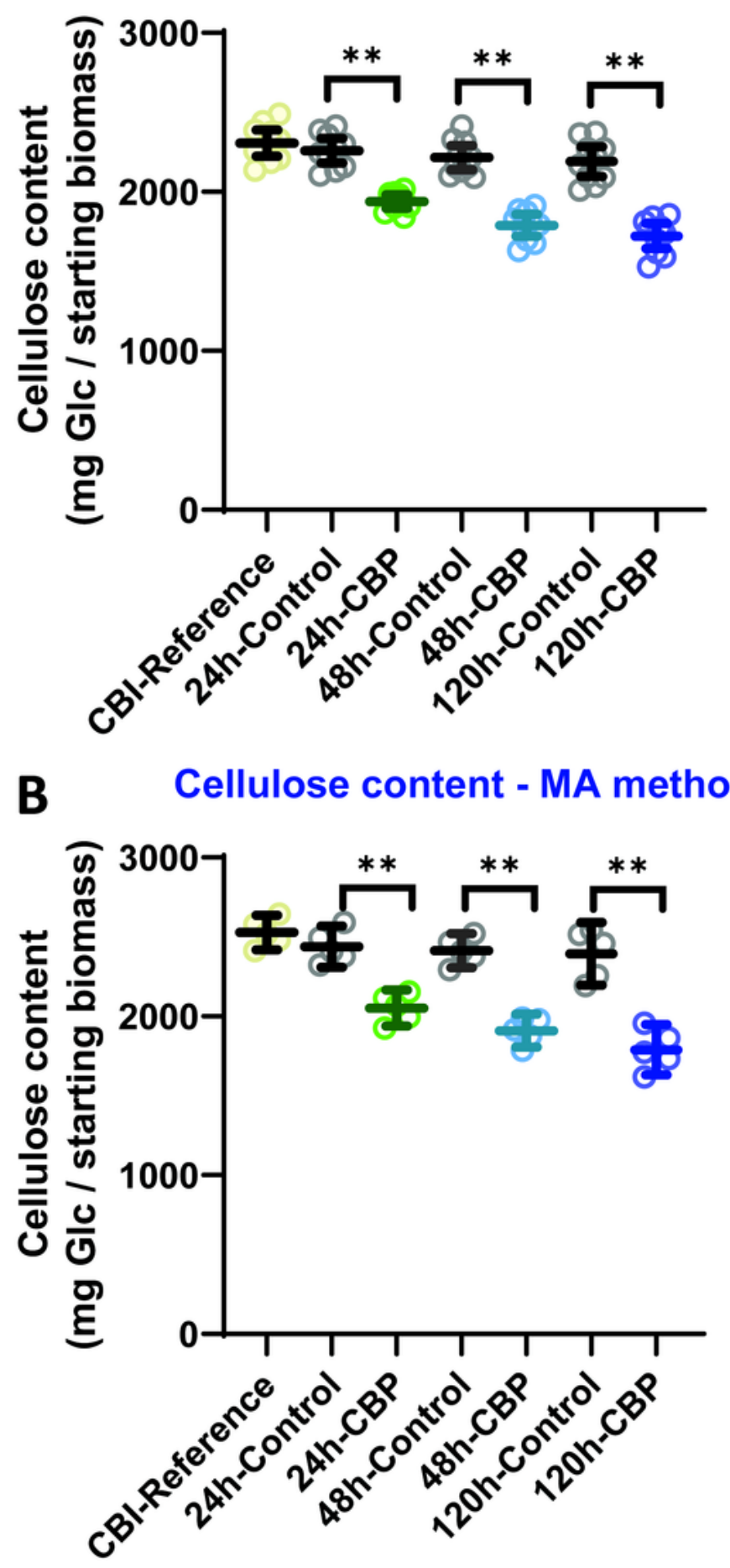

Figure 5

Cellulose content of solid residues recovered during $\mathrm{C}$. thermocellum fermentation and in fermentation controls. Cellulose content of alcohol insoluble residue (AIR) (i.e. cell walls) of solid residue recovered after consolidated bioprocessing and in fermentation controls by (A) Saeman hydrolysis $[19,20]$ and (B) 

average $\mathrm{mg}$ of glucose per starting biomass $(5.68 \mathrm{~g})$. Mean \pm SD of 2 biological and three technical replicates $(n=6)$. P value is ** $P \leq 0.01$ at significant level.

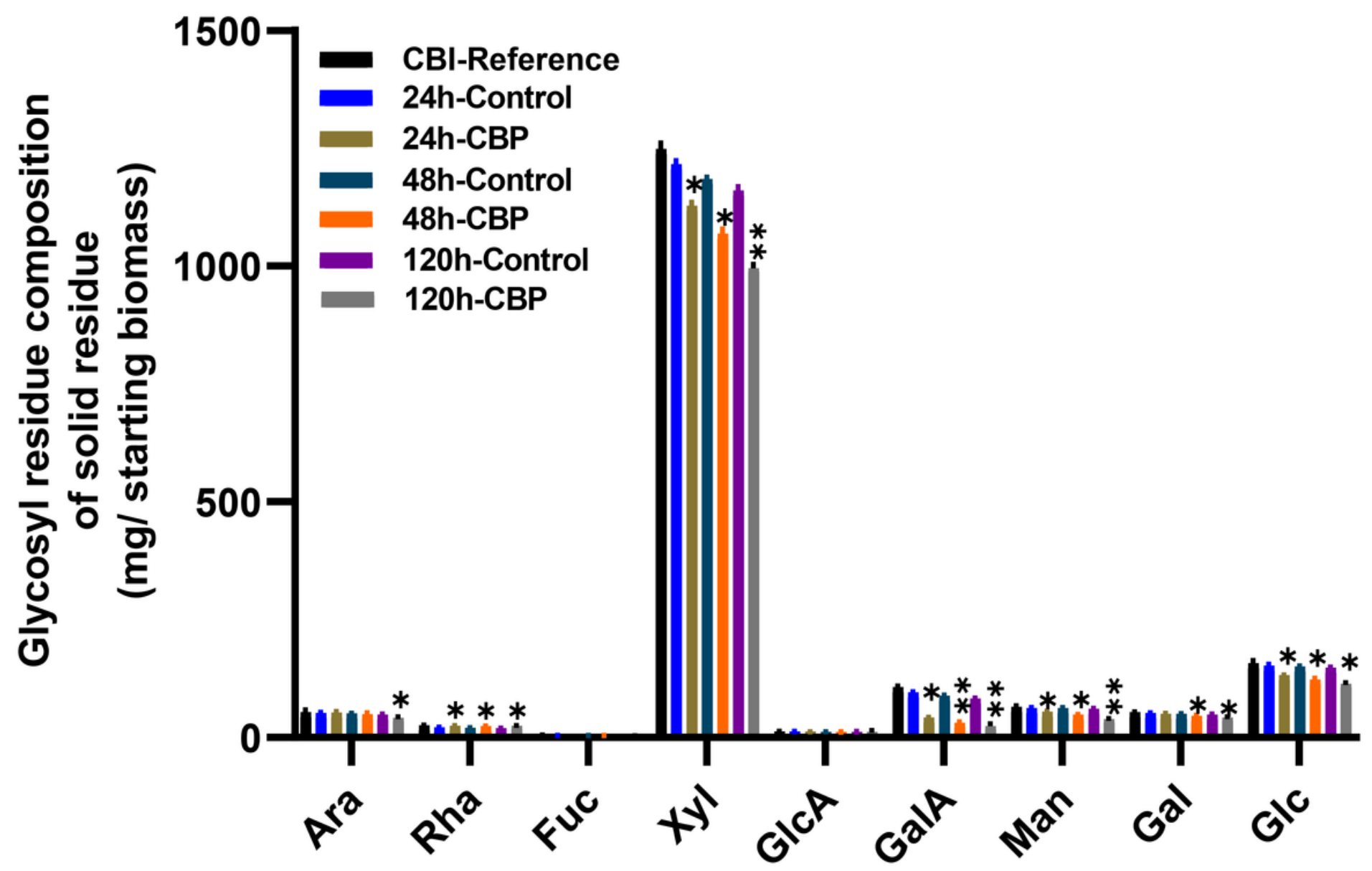

Figure 6

Glycosyl residue composition by trimethylsilyl (TMS) derivatization and GC-MS of AIR (alcohol insoluble residues) from solid residues recovered after fermentation of CBI reference poplar biomass by $\mathrm{C}$.

thermocellum. The amounts of sugar are represented as average $\mathrm{mg}$ of $5.68 \mathrm{~g}$ starting biomass. Mean \pm SD of two biological and two technical replicates $(n=4)$. Stars indicate values that are different at * $P \leq$ 0.05, ** $P \leq 0.001$ significant level. 

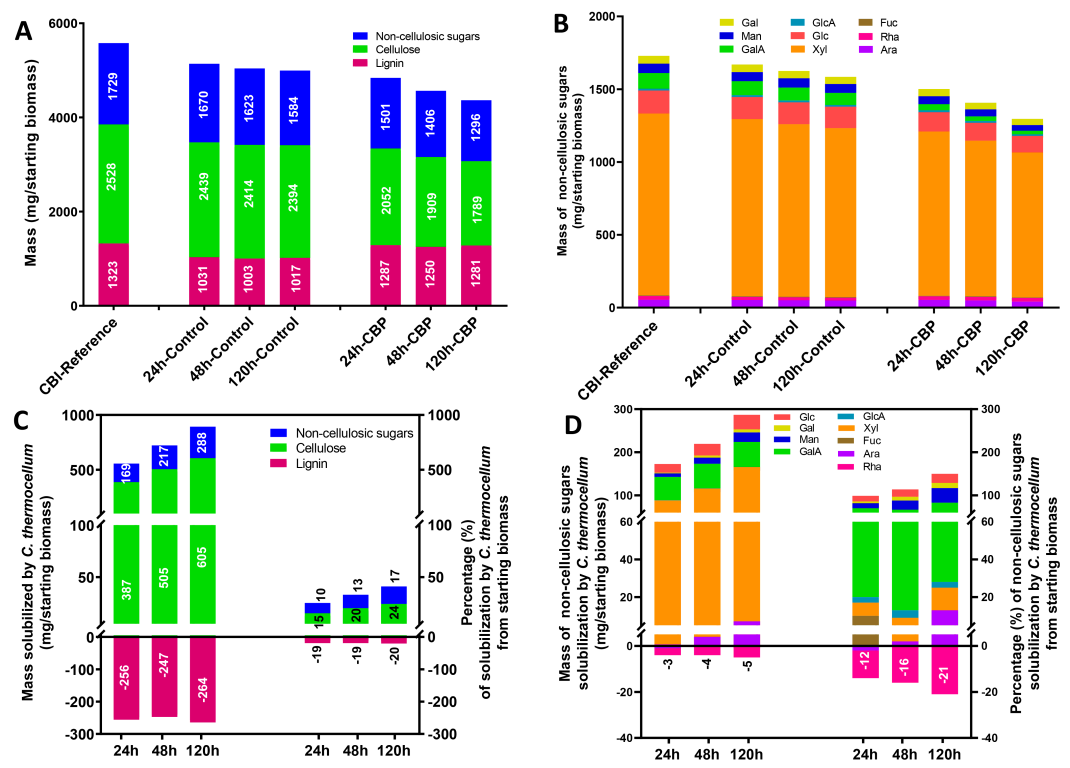

Total mass and solubilization of lignin, cellulose, and non-cellul osic sugars from solid residue after CBP fermentation of $5.68 \mathrm{~g}$ starting milled poplar biomass. (A) Mass (mg/starting material) of total lignin, cellulose, and non-cellulosic sugars remaining in the solid residue. These data are from Fig. 4, Fig. 5, and Fig. 6. (B) Mass (mg/starting material) of total non-cellulosic sugars (Xyl, Glc, Man, Ara, Gal, Rha, Fuc, GalA, GlcA) remaining in the solid residues at the times indicated. The mass of total monosaccharide is presented as a stacked bar from data from Fig. 6. (C) Mass (left) and percentage (right) of non-cellulosic sugars, cellulose and lignin solubilized by $C$. thermocellhum. All solubilization calculations were done based on the starting biomass. Negative solubilization indicates that this component accumulates in the solids. Detailed calculations are as follows:

Mass of polymer solubilized during control fermentation (without microbe) $=\mathrm{A}$

$A=$ mass $(\mathrm{mg})$ of sugar polymer in starting bi omass before CBP minus the mass of solids in 24 $\mathrm{h}, 48 \mathrm{~h}$, and $120 \mathrm{~h}$ fermentation control.

Mass of polymer solubilized during the CBP process (i.e. solubilized by the process conditions plus solubilization by $C$. thermocell $2 a m)=B$

$\mathrm{B}=$ mass (mg) of polymer in starting biomass before CBP minus the mass of solids in $24 \mathrm{~h}, 48 \mathrm{~h}$, and $120 \mathrm{~h} \mathrm{CBP}$ fermentations)

Mass of polymer solubilized by $\mathrm{C}$. thermocellum $=\mathrm{B}-\mathrm{A}$.

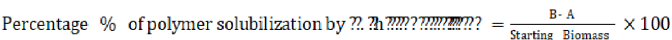
(D) Mass and percentage of non-cellulosic sugars solubilized by $C$. thermocellum. Both mass and percentage of solubilization are calculated as described in Table 1. 24h: Glc (13\%), Gal (4\%), Man (12\%), GalA (50\%), GlcA (3\%), Xyl (7\%), Fuc (10\%), Rha (-12\%), Ara (-2\%), 48 h: Glc (17\%), Gal (9\%), Man (21\%), GalA (54\%), GlcA (4\%), Xyl (9\%), Fuc $(0 \%)$, Rha (-16\%), Ara (2\%). 120 h: Glc (21\%), Gal (12\%), Man (34\%), GalA (55\%), GlcA (3\%), Xyl (13\%), Fuc (12\%), Rha (-21\%), Ara (13\%). Mass values are given in Table 1.

\section{Figure 7}

See image above for figure legend

\section{Supplementary Files}

This is a list of supplementary files associated with this preprint. Click to download.

- FigureS1.tif

- Figures2.tif

- Figures3.tif

- FigureS4.tif

- FigureS5.tif

- FigureS6.tif

- Figures7.tif 